\title{
Molecular changes in the multistage pathogenesis of head and neck cancer
}

\author{
Brian J. Park ${ }^{a}$, Simion I. Chiosea ${ }^{c}$, and Jennifer R. Grandis ${ }^{b, d,}{ }^{*}$ \\ aDepartment of Otolaryngology, Massachusetts Eye and Ear Infirmary, Boston, MA, USA \\ ${ }^{b}$ Department of Otolaryngology, University of Pittsburgh Cancer Institute, University of Pittsburgh, \\ Pittsburgh, PA, USA \\ 'Department of Pathology, University of Pittsburgh Cancer Institute, University of Pittsburgh, \\ Pittsburgh, PA, USA \\ ${ }^{d}$ Department of Pharmacology, University of Pittsburgh Cancer Institute, University of Pittsburgh, \\ Pittsburgh, PA, USA
}

\begin{abstract}
Head and neck squamous cell carcinomas (SCCHN) arise in the mucosa of the upper aerodigestive tract at multiple anatomic sites. While tobacco and alcohol exposure remain the primary risk factors for this malignancy, infection with the human papilloma virus is emerging as a major contributing factor to cancers that arise primarily in the oropharynx. Despite therapeutic advances, survival has remained relatively unchanged over the past few decades. Increased understand of the cellular and molecular biology of these cancers will improve our understanding of this malignancy and facilitate the development of more effective therapeutic strategies. Alterations that have been studied to date include genetic and epigenetic changes. While the epidermal growth factor receptor (EGFR) is the only established molecular therapeutic target, other proteins and pathways are under active investigation to determine their contribution to SCCHN carcinogenesis and progression.
\end{abstract}

\section{Keywords}

Head and neck cancer; molecular pathogenesis; squamous cell carcinoma; leukoplakia; premalignant; human papilloma virus

\section{Introduction}

Head and neck cancer includes a heterogeneous group of malignancies involving the oral cavity and oropharynx, nasopharynx, hypopharynx, larynx, nasal cavity and paranasal sinuses, ear, salivary glands and thyroid. Squamous cell carcinoma of the head and neck (SCCHN) represents more than $90 \%$ of all head and neck cancers and arises from the mucosa of the upper aerodigestive tract. The worldwide incidence of SCCHN exceeds half a million cases annually, making it the sixth most frequent cancer globally. In the United States, 47,560 cases are expected to occur in 2008 , accounting for only about $3 \%$ of all cancers nationwide [1]. The age-adjusted incidence rates of SCCHN in the US have steadily declined since the 1980s, primarily due to diminished smoking rates. Increased tobacco use in developing countries indicates that an increased incidence of SCCHN cases may be

\footnotetext{
(C) 2011 - IOS Press and the authors. All rights reserved

"Corresponding author: Jennifer R. Grandis, M.D., 200, Lothrop Street, Eye and Ear Institute Building, Suite 500, Pittsburgh, PA 15213, USA. grandisjr@upmc.edu.
} 
subsequently observed [2,3]. Despite advances in improved diagnosis and multimodality therapies, however, survival rates have remained relatively unchanged and half of all individuals with SCCHN ultimately succumb to their disease (SEER).

The most significant risk factors for developing SCCHN remain tobacco and alcohol use. Oncogenic human papillomavirus (HPV) is now considered a causative agent in certain SCCHN subsites, specifically the oropharynx. Other risk factors associated with this disease include dietary deficiencies, poor oral hygiene, asbestos and indoor air pollution from fossil fuel combustion [4]. Understanding the evolving molecular pathophysiology of head and neck tumorigenesis is vital for the development of more effective preventative, diagnostic, and treatment strategies.

It has been established that SCCHN arises from a multistage pathogenesis process similar to that reported in the genetic progression model for colon cancer [5]. Molecular alterations in epithelial cells generally precede phenotypic histologic changes and accumulate throughout malignant transformation from the benign to premalignant and invasive states. Increased understanding of the cellular and molecular biology of solid tumors has further improved the surgical pathologist's diagnostic and prognostic ability to assess SCCHN tumors. For example, the identification of both known and potential tumor markers including chromosomal changes, oncogenes, and tumor suppressor genes has far-reaching implications to accurately predict the biologic behavior of any given tumor and its response to therapy. This chapter will review the molecular alterations identified to date, as well as potential applications to diagnose and treat SCHNN in its early stages before the development of advanced locoregional disease and distant metastasis.

\section{Alterations in premalignant oral lesions}

Field cancerization is a potential mechanism for SCCHN tumorigenesis. Many patients diagnosed with SCCHN have had chronic exposures to tobacco and alcohol and are at increased risk of local recurrence and second primaries. In 1953, Slaughter et al. described the mucosa of the upper aerodigestive tract of such patients to be "condemned" since large areas are chronically exposed to the environmental mutagens and thus, are all at risk for developing dysplasias, and subsequently, carcinomas [6]. Such phenomenon can provide a pathogenetic basis for the occurrence of synchronous and metachronous primaries.

In 1990, Vogelstein et al. was the first to propose a multistage pathogenesis for cancer, specifically of the colon. This genetic model for colorectal tumorigenesis included the activation of oncogenes and inactivation of tumor suppressor genes. He described that multiple "hits" (at least four mutations) were necessary for malignant transformation. Stepwise advances have been suggested including initiation, promotion and progression. This schema has been observed in many other cancers including those of the brain, bladder, in addition to head and neck. Califano et al. reported a "Vogelgram" for SCCHN, which linked histologic features in the progression of SCCHN to specific molecular alterations [7]. They proposed that it is the accumulation of genetic events, not the order, that determines progression.

Further investigation focused on the transcriptional changes that occur in the progression from normal-appearing mucosa to dysplastic tissue and invasive SCCHN [8]. Microarray analysis of RNA isolated from a continuum of such specimens identified genes with differential expression patterns. Similarly to the genetic progression model of SCCHN, this group showed that most of the alterations in their transcriptional progression model occurred before the development malignancy. Identification of genes that were significantly upregulated included integrin $a 6$. 


\subsection{Cytogenetic alterations}

Genomic changes that occur in premalignant and malignant lesions can manifest themselves on different levels (chromosomal, DNA, RNA, and protein) and in a variety of ways such as point mutations, amplifications, deletions, and chromosomal alterations. Common methods to screen for the presence of these changes include, but are not limited to, conventional cytogenetics, comparative genomic hybridization (CGH), spectral karyotyping, and cDNA microarrays. CGH is a cytogenetic technique that assesses for gains or losses in DNA content (e.g., chromosomal imbalances) within a tumor's entire genome. Tumor DNA can be obtained by microdissection of an H\&E-stained specimen, while DNA from peripheral blood leukocytes serves as a control. CGH is performed by hybridizing the tumor DNA and normal DNA, which are labeled with different fluorescent tags, to normal human metaphase preparations. Epifluorescent microscopy and image analysis is employed to detect DNA gains or losses as compared to controls. CGH does not show structural changes between chromosomes. The minimal size of chromosome that can be used is similar to conventional cytogenetics $(3-5 \mathrm{Mb})$.

Weber et al. employed this technique to determine the average number of chromosomal imbalances in 12 oral premalignant lesions (including dysplasias and carcinomas-in situ) and 14 invasive oral SCCHNs [9]. An average of $3.2 \pm 1.2$ imbalances were seen in premalignant lesions while invasive SCCHNs had a significantly higher average of $11.9 \pm$ $1.9(p=0.003)$ imbalances. Within the premalignant lesions, gains were identified on $8 \mathrm{q}$ and $16 p$ while losses were found on 3p, 5q, 13q, and 4q, 8p, and 9p. In individual biopsies from the same subject that contained both premalignancy and invasive carcinoma, most of the genomic alterations found in premalignancy were also found in SCCHN.

Brieger et al. used CGH to analyze chromosomal alterations in 22 oropharyngeal SCCHN tumors and their surrounding benign mucosa [10]. In the morphologically benign mucosa collected $2 \mathrm{~cm}$ from the primary tumor, no chromosomal alterations were identified. In benign mucosa located $1 \mathrm{~cm}$ from the tumor, the most common amplifications were in $15 \mathrm{q}$ and 21q. Nearly all of these changes were seen in the primary tumor as well. The authors speculated that some second primaries may be explained by a monoclonal model followed by lateral epithelial spread. Kim et al. utilized chromosome in-situ hybridization (CISH) targeting chromosomes 9 and 17 to characterize oral lichen planus lesions [11]. When mucosal epithelium was compared to control lymphocytes, a higher proportion of polysomic and monosomic cells for chromosome 9 was found $(p=0.0017)$.

These cytogenetic findings are consistent with previous reports implicating molecular changes early on in head and neck tumorigenesis before histologic and phenotypic changes, in addition to their accumulation through successive stages. CGH continues to be an invaluable tool, but the other aforementioned techniques are also widely used.

\subsection{Molecular alterations}

2.2.1. Microsatellite instability-Microsatellites are repeats of non-coding DNA sequences that occur normally within the human genome. Defects in the DNA repair process can lead to microsatellites that are abnormally short or long; this process has been termed microsatellite instability (MI). MI is indirect evidence of an abnormal mismatch repair (MMR) protein's function (hMLH1, PMS2, MSH2, MSH6). A proposed mechanism relevant in SCCHN tumorigenesis is through promoter hypermethylation. When MMR promoters are hypermethylated, it provides indirect evidence of a higher chance that promoters of tumor suppressor genes are hypermethylated too, and therefore nonfunctional [12]. Alternatively, when a microsatellite repeat replication error goes uncorrected, a germ line hereditary mutation could result leading to inactivation of tumor suppressor genes and 
uncontrolled cell and tumor growth. This concept of a mutator phenotype provides an alternative to a multistage accumulation of genetic alterations to explain head and neck tumorigenesis. Specifically, the loss of function of a gene critical for the repair of DNA damage greatly increases the mutation rate at other loci leading to genome-wide instability.

In a study of 93 premalignant and 18 invasive SCCHN cases, an increasing trend of MI was found from hyperplasias (6\% of specimens) to dysplasias/CIS (27\%) and to invasive cancers (33\%) [13]. A similar trend was found in another study where $15 \%$ of dysplasias and $30 \%$ of invasive cases manifested MI at multiple loci [14]. Partridge et al. found as high as 55\% of 31 leukoplakias and erythroplakias to show MI [15]. The incidences of MI found in these and other studies in head and neck malignancies are significantly higher than those reported in breast, skin and non-small-cell lung cancers. The prevalence of MI appears to vary between tumor types.

2.2.2. Loss of heterozygosity ( $\mathrm{LOH})$-An allele of a gene, such as a tumor suppressor gene, can be inactivated by mutation. When this occurs in a parent's germline cell, the inactivated allele is passed onto its offspring resulting in heterozygosity. When genomic loss occurs in the somatic cell of the offspring affecting the remaining allele, $\mathrm{LOH}$ occurs and tumor suppressive function in that cell is lost. $\mathrm{LOH}$ assays commonly employ microsatellite analysis to assess polymorphic chromosomal regions that map in or near tumor suppressor genes.

It is known that $\mathrm{LOH}$ at 9p21 and/or 3p14 in premalignant oral lesions increases their probability of malignant transformation [16]. Weber et al. identified LOH at 3p, 9p, 17p, and 18q in dysplasias and 11q, 13q, and 14q in CIS [9]. Other chromosomal losses have been associated with increased risk: 4q, 8p, 11q, 13q, and 17p. Hyperplastic or dysplastic lesions with $\mathrm{LOH}$ at $3 \mathrm{p}$ and/or 9p plus one of the other above losses were found to have a 33-fold increase in cancer risk [17]. In lesions that lack significant dysplasia to guide estimation of cancer risks, molecular markers such as these may yet prove to be useful. Zhang et al. proposed a staging system incorporating assessments from both histology and LOH criteria [18].

2.2.3. p53 - p53 is a well-characterized tumor suppressor gene located on chromosome $17 \mathrm{p}$. Its function is to regulate cell growth arrest and apoptosis. The p53 protein has a very short halflife, and thus, is difficult to detect in benign tissues. Overexpression of p53 can result from mutation, a defect in its degradation, or from binding to other proteins. On the whole, mutations of the p53 tumor suppressor gene have been found in about half of SCCHN tumors and are the most common genetic alteration found in human malignancy. The types of mutations vary including point mutations, transversions, transitions, and deletions. The most common type are missense mutants, which have a longer halflife than the wild-type form. Various proteins are known to bind with p53 such as SV40 large T, which blocks its DNA binding capability. Binding with adenovirus E1B blocks p53's transcriptional activity. Finally, binding with HPV E6 targets p53 for accelerated degradation.

During progressive steps in SCCHN carcinogenesis, increasing frequencies of p53 alterations and genomic instability have been identified. Shin et al. analyzed p53 expression and chromosomal polysomy via immunohistochemistry and chromosome insitu hybridization, respectively, in 136 epithelial specimens. $19 \%$ of adjacent normal-appearing mucosa, $29 \%$ of hyper-plastic lesions, $46 \%$ of dysplastic lesions, and 58\% of SCCHN tumors expressed p53. Normal-appearing mucosa lacked detectable p53 levels as expected. p53-positive dysplastic lesions showed a 2 to 4-fold increase in the level of chromosome 9 and 17 polysomy ( $p=0.002$ and 0.005 , respectively). In the remaining groups, increasing 
trends were found with histological progression [19]. These findings suggest that premalignancy is associated with altered p53 expression and increased genomic instability, which may be early markers of carcinogenesis.

A Dutch study attempted to correlate p53 staining patterns with risk of malignant transformation. Thirty-five premalignant lesions were followed for a range of 1-16 years. Staining patterns included suprabasal or basal expression or no expression at all. The positive predictive value of suprabasal p53 staining progressing into carcinoma was $86 \%$ whereas the negative predictive value of basal or absent p53 expression was 82\% [20].

The implications of p53-positivity in histologically normal-appearing mucosa are incompletely understood. Some attribute it as an effect of field cancerization; however, it is unknown whether any prognostic significance is associated with this finding. Ogden et al. examined specimens from 13 patients with p53-positive normal-appearing mucosa and compared their rate of second primaries to those of 9 patients with p53-negative normalappearing mucosa [21]. No significant difference was seen between the groups after a mean follow-up of 4.75 years and 4.1 years, respectively.

2.2.4. Retinoic acid receptor- $\beta$-Retinoids (natural and synthetic derivatives of vitamin A) regulate cell growth and differentiation and have growth-suppressive effects in epithelial cells. Retinoic acid receptor- $\beta$ (RAR- $\beta$ ) is a steroid hormone receptor whose expression is suppressed in premalignant and malignant head and neck lesions via an unknown mechanism. Restoration of RAR- $\beta$ expression, e.g., with isoretinoin or 13-cis retinoic acid, seems to correlate with the growth-inhibitory effect of retinoids in solid tumors and premalignancies. However, as will be discussed later, isoretinoin failed to show efficacy as monotherapy in chemoprevention and is being studied in combination with other biologic agents (see Section 5.2. Biochemoprevention).

2.2.5. Reactivation of telomerase-Telomeres are DNA repeats that cap the ends of chromosomes for stability purposes. The enzyme telomerase replenishes telomeres as they necessarily get consumed by each replicative cycle. Telomerase has been detected in germline cells, which allows them to divide repeatedly. However, most postnatal somatic cells express it only at very low levels in a cell-cycle dependent manner. Increased telomerase activity is usually associated with longer cell survival. Also, since it is activated in many human cancers and is undetectable in most normal tissues, telomerase makes for an attractive potential tumor marker.

Patel et al. utilized a TRAP (telomerase rapid amplification protocol) assay to identify telomerase activation in $78 \%$ of 110 SCCHN tumors and $85 \%$ of 36 precancerous lesions [22]. 53\% of adjacent normal-appearing tissues ( $1 \mathrm{~cm}$ away from tumor) were also positive. Additionally, mean telomere length was shorter in malignant tissues as compared to adjacent normal-appearing tissues. Patients with telomerase activation in adjacent normal-appearing tissues and patients with higher telomere length in malignant tissues were noted to have poor disease-free survival. Similar to $\mathrm{LOH}$, telomerase activation appears to be an early event in carcinogenesis.

Liao et al. used a modified TRAP assay to identify telomerase activity in 39\% of normalappearing mucosal samples, 55\% of leukoplakias, and 82\% of oral SCCHN samples [23]. Zhang et al. developed a reproducible liquid scintillation counter to detect low radioactive counts in all normal-appearing samples and high counts in $86 \%$ of malignant head and neck tumors. In ten dysplastic specimens, two with severe dysplasia showed higher telomerase activities than those with mild dysplasia [24]. Sumida et al. also used a modified TRAP assay to detect telomerase activity in $71 \%$ of dysplastic lesions. RTPCR was employed to 
measure the expression levels of the three telomerase components-hTR, TP1, and hTRT. Detection of hTRT-mRNA by RT-PCR appeared to be more sensitive for telomerase than measurement by the TRAP assay [25].

2.2.6. $\mathbf{p} 16 /$ cyclin $\mathrm{D} 1 / \mathbf{R} \mathbf{b}$ - In addition to $\mathrm{p} 53, \mathrm{Rb}$ is the other major tumor suppression pathway in human carcinogenesis. Rb normally suppresses cell growth by binding to E2F1 transcription factors and preventing cells from advancing from $\mathrm{G} 1$ into $\mathrm{S}$ phase of the cell cycle (G1 arrest). The proto-oncogenes, cyclin D-dependent kinases CDK4 and CDK6, phosphorylate and inactivate $\mathrm{Rb}$ thereby allowing for cell cycle progression. Cyclin $\mathrm{D} 1$ facilitates CDK complex formation and pRb binding. p16 ${ }^{I N K 4 a}$ is one type of cyclindependent kinase (CDK) inhibitor, which binds to CDK4 and CDK6, thereby activating $\mathrm{Rb}$ and G1 arrest. The major control point of p16 is in the methylation of its promoter. Another CDK inhibitor class includes $\mathrm{p} 21^{\text {waf- }-1}$, whose role also includes inhibiting DNA replication and inducing cell cycle arrest. Altered expression of pRb and p16 has been reported in oral carcinomas [26]. In fact, alterations in p16 are considered the most common genetic alteration in SCCHN [27]. Cyclin D1 amplification and overexpression have been also identified [26]. The HPV oncoprotein E7 is known to inactivate Rb.

Soni et al. performed IHC analysis of p16, cyclin D1, and Rb expression in 81 normalappearing oral mucosal specimens, 52 hyperplastic and 38 dysplastic lesions and 220 SCCHN tumors. $11 \%$ of hyperplastic and dysplastic and $18 \%$ of SCCHN tumors revealed alterations in all three genes. Loss of p16 was identified as the earliest event. Loss of Rb was associated with transition from hyperplasia to dysplasia. Rb loss with cyclin D1 overexpression or p53 overexpression alone was associated with transition to SCCHN. Loss of $\mathrm{Rb}$ along with $\mathrm{p} 53$ overexpression was a significant predictor of survival.

2.2.7. Mitochondrial DNA-As opposed to alterations in human genomic DNA, changes can occur in mitochondrial DNA instead. A common location for polymorphisms and mutations is the poly-cytosine tract (C-tract) of the displacement loop of the mitochondrial genome. Ha et al., tested 137 premalignant lesions for C-tract DNA alterations using PCR and polyacrylamide gel electrophoresis [28]. When compared to controls, 37\% of patients exhibited C-tract alterations. Moreover, the occurrence of $\mathrm{C}$-tract changes increased with increasing severity of dysplasia up to carcinoma in-situ. An identical pattern was seen in 8 out of 10 patients with multiple, synchronous tumors as well as in 2 of 3 patients with metachronous tumors. These findings suggest that $\mathrm{C}$-tract alterations may be markers of clonality when multiple tumors occur within the same patient.

2.2.8. EGFR/STAT3-The epidermal growth factor receptor (EGFR) pathway has been investigated due its role in SCCHN tumorigenesis. Epidermal growth factor receptor (EGFR) and its primary autocrine ligand, transforming growth factor- $a$ (TGF- $a$ ), are upregulated early during carcinogenesis via gene amplification and/or transcriptional activation. Overexpression of EGFR is present in malignant, premalignant and normalappearing tissues from SCCHN patients and is correlated with poor prognosis [29,30]. Staining of TGF- $a$ in the statum germinativum increased linearly in oral leukoplakia with mild, moderate, and severe dysplasia as compared to control mucosa. EGFR staining increased linearly in the stratum spinosum in oral leukoplakia with increasing degrees of dysplasia [31].

Upon binding of ligand, EGFR dimerizes and its intracellular tyrosine kinase (e.g., JAK2) activates and phosphorylates several downstream molecules, one of which is the STAT3 transcription factor. STAT3 contributes to a diverse array of cellular responses, including proliferation, differentiation, and suppression of apoptosis. Phosphorylated STAT3 dimerizes and translocates from the cytoplasm to the nucleus. In the nucleus, the 
phosphorylated/activated STAT3 dimer binds to STAT3 DNA response elements in the promoters of target genes, stimulating their transcription and gene expression. It is the repertoire of STAT3 target genes that are induced in a particular cell type helps to determine the cellular response to growth factor and cytokine stimulation. Overexpression of EGFR is also correlated with constitutive activation of STAT3 via an antiapoptotic mechanism (e.g., Bcl- $x_{L}$ ). Inhibition of STAT3 via anti-sense strategies increases apoptosis and decreases $\mathrm{Bcl}-\mathrm{x}_{L}$ expression in a xenograft model [30]. Early activation of EGFR and STAT3 in carcinogenesis implicates this pathway as potential therapeutic target in premalignancy.

\section{Alterations in invasive squamous cell carcinoma}

\subsection{Cytogenetic alterations}

Many of the molecular alterations found in SCCHN are caused by cytogenetic changes. Conventional cytogenic analysis involves preparation of metaphase spreads of cultured tumor biopsies. Individual chromosomes can be analyzed with light microscopy after Trypsin-Giemsa banding. The entire genome may be analyzed for structural and numeric chromosomal changes in individual cells. Intratumoral heterogeneity can thus be studied.

The most frequent cytogenetic alterations in SCCHN include chromosomal gains on 3q, 8q, 9q, 20q, 7p, 11q13, and 5p. Genomic losses are more frequent than gains and have been identified on 3p, 9p, 21q, 5q, 13q, 18q, and 8p [32]. Investigation of these alterations in untreated head and neck specimens enables the identification of genes responsible for disease phenotypes and also improves the understanding of the pathophysiology behind the disease. Conventional cytogenetics of head and neck tumors has been difficult due to low mitotic index, small specimen size and difficulties in cell culturing. Both classical and molecular cytogenetic techniques have shown SCCHN to have a complex karyotype with the most common change being tetraploidization. Moreover, an average number of 15 aberrations are found across the genome [33]. These alterations can include deletions, translocations and isochromosomes, which constitute the most common mechanism in the case of SCCHN.

3q gains are among the most common alterations in SCCHN. The AIS (p40/p73L) gene maps to distal $3 q$ and is an important candidate marker. $7 p$ gains (7p12-p22) are seen in onehalf to two-thirds of SCCHN cell lines in the form of copy number increases. EGFR is located on $7 \mathrm{p} 12$ and has been associated in separate reports with increased copy number and increased gene/protein expression. 8q gains are associated with copy number increases with amplification of the MYC oncogene in 24\% of 34 SCCHN cell lines studied. 11q13 amplifications involve cyclin D1 (CCND1) and are found in 30-50\% of tumors. These amplifications have been correlated with poor prognosis by CGH [34]. As mentioned previously, $3 p$ loss is identified as the most common genomic loss. The FHIT (fragile histidine triad) gene maps to 3p14.2 in 26 SCCHN cell lines. This gene encodes for an enzyme involved in purine metabolism, which can be disrupted during translocations or losses that affect chromosome 3 . The common loss at the 9p21 loci is thought to include the p16/CDKN2A gene. The loss of 18q by cytogenetics has been associated with poor clinical outcome [35].

CGH has been useful to elucidate cytogenetic differences amongst SCCHN tumors from different anatomic subsites. Tumor biology and clinical prognosis may vary by anatomic subsite. Patmore et al. applied CGH techniques to assess for chromosomal aberrations in 68 SCCHN tumors of the larynx, hypopharynx, and oropharynx [33]. Laryngeal tumors had a statistically significant increase in aberrations when compared to other tumors. This subsite was also significantly associated with $-4 \mathrm{p},+8 \mathrm{q},+12 \mathrm{q}$, and $-18 \mathrm{q}$ when compared with other subsites. Hypopharyngeal tumors were associated with $2 \mathrm{q}$, while no associations were found 
in oropharyngeal tumors. Although the laryngeal findings were consistent with other published reports, it is unclear why more genetic aberrations were not found in the hypopharyngeal group. For example, Juhasz et al. reported more aberrations in hypopharyngeal, compared to laryngeal, tumors [36]. The former authors speculated the potentially less complex karyotypic changes associated with oncogenic HPV-associated tumors as a reason for the more complex laryngeal findings.

Molecular alterations are also found in non-squamous cancers of the head and neck. The most specific mucoepidermoid carcinoma-associated genetic alteration is the $t(11 ; 19)$ (q21;p13) translocation initially identified by traditional karyotyping [37]. This translocation generates a mucoepidermoid carcinoma translocated 1 (MECT1)-mastermind-like 2 (MAML2) gene fusion product consisting of exon 1 of MECT1 fused to exons 2-5 of MAML2 [38]. Patients with fusion-positive tumors are generally younger, have lower grade tumors, and a significantly lower risk of local recurrence, metastasis or tumor-related death. Only a few translocation-positive aggressive high-grade MEC have been recently described [39].

\subsection{Molecular alterations}

3.2.1. p53-Early molecular studies failed to identify a correlation between $\mathrm{p} 53$ expression and survival of SCCHN patients. When patients with laryngeal primaries were selected for, positive p53 expression was significantly associated with poor patient outcome [40,41]. In studies focusing on certain types of mutations (e.g, missense, nonsense), correlations were observed with survival $[42,43]$. Other studies between p53 mutations and prognosis have yielded conflicting data $[44,45]$. In a recent multicenter study, Poeta et al. analyzed tumor DNA for p53 mutations in 560 SCCHN patients via Affymetrix gene chips and DNA endonuclease/high-performance liquid chromatography [46]. Only patients undergoing primary surgery with curative intent were included. p53 mutations, and particularly disruptive p53 mutations, correlated with decreased survival (with a hazard ratio of 1.7 for disruptive mutations).

The prevalence of p53 alterations in head and neck cancers underscores its importance in tumorigenesis. Inactivation of this tumor suppressor gene has been correlated with resistance to chemotherapy [47]. Restoration of function via intratumoral injection of adenoviral p53 gene therapy (Ad-p53 or INGN-201) has shown favorable results in phase I and phase II trials in patients with advanced and recurrent SCCHN [48]. Phase III trials are pending comparing INGN 201 with IV methotrexate and comparing INGN 201 and standard chemotherapy with chemotherapy alone.

3.2.2. Telomerase activation-Lee et al. measured telomerase activity via TRAP in the peripheral blood mononuclear cells of 120 patients-100 with SCCHN and 20 controls [49]. Telomerase positivity significantly correlated with higher T stage $(p=0.005)$; higher $\mathrm{N}$ stage $(p=0.002)$; and higher AJCC stage $(p<0.001)$. Multivariate analysis showed telomerase expression to be an independent predictor of survival. $(p=0.017)$. This is the first time that the expression of a protein in peripheral blood cells from SCCHN patients demonstrated prognostic potential. Thurnher et al. used a modified semiquantitative TRAP assay to assess 16 SCCHN tumors. When stratified by the presence or absence of cervical metastases, a statistically significant difference in telomerase activity was seen between the two groups [50]. Although the sample size was limited, this study shows preliminary feasibility in using biomarkers such as telomerase to identify patients at high risk of cervical metastasis. This would have dramatic effects in outcomes by selectively matching patients with more aggressive therapy. 
3.2.3. HPV-There is increasing evidence that suggests the association of high-risk HPV strains with a subset of head and neck cancers is causal. Viral DNA is found in CIS, primary SCCHN tumors and metastases by a variety of molecular techniques (e.g. Southern blot, PCR, in situ hybridization). In fact, DNA has been found in as many as 50\% of oropharyngeal cancers, particularly in nonsmokers. Viral oncoproteins E6 and E7 of highrisk HPV strains 16 and 18 disrupt the p53 and Rb tumor suppressor pathways, respectively, thereby providing potential mechanisms for transformation. However, methods that detect HPV (e.g., rinses for oral cavity tumors) in patients do not necessarily signify host integration by HPV. These cases, therefore, may not contribute to disease phenotype. When HPV is present in the cell, some investigators differentiate between episomal (p16 IHCnegative; p16 being a surrogate HPV marker) and integrated (p16 IHC-positive) forms of HPV infection. HPV positivity in normal adjacent tissues can be considered a rare occurrence. In a recent survey of 200 normal tonsils, the prevalence of HPV 16 and 18 was found to be zero [51,52]. Whether HPV positivity equals infection remains to be clearly defined and further investigation is warranted. Cancers that are tested for the presence of HPV mRNA should be assessed for specific serotypes.

HPV-positive tumors have the following characteristics: most commonly originating in the oropharynx (tonsil or tonguebase), basaloid histology, less or no tobacco or alcohol history, and improved survival when compared to HPV-negative tumors [53]. Additionally, there has been a rise in oropharyngeal cancer incidence over the past 30 years detected in adults younger than 45 years of age [4]. HPV involvement has implications for the potential transmission of some SCCHN malignancies via oro-genital contact and the potential application of HPV vaccines targeted at males and females at risk.

3.2.4. EGFR/STAT3-Epidermal growth factor receptor (EGFR) represents a validated therapeutic target in SCCHN. Cetuximab is a chimeric monoclonal antibody that binds to the extracellular domain of EGFR. Several phase II trials reported on the efficacy of cetuximab and platinum-based chemotherapy in recurrent and refractory SCCHN cases [54,55]. Responses rates in patients with progressive disease were approximately $10 \%$ with the combination therapy. The severity of one of the side effects, acneiform rash, was directly proportional to outcome and survival. The phase III trial conducted by Burtness et al. found increased response rates for recurrent/metastatic SCCHN patients treated with cisplatin and cetuximab (26\%) vs. cisplatin and placebo $(10 \%)(p=0.03)$ [56].

In 2006, Bonner et al. conducted a phase III trial of cetuximab plus radiotherapy for patients with locoregionally advanced SCCHN vs. radiotherapy alone [57]. An unquestionable improvement was found in locoregional control, progression-free survival, and overall survival in the cetuximab and radiotherapy group. Surprisingly, the addition of cetuximab did not increase severe mucositis rates. Although the radiotherapy schemes were not uniform throughout, the improvements seen in outcomes without a significant increase in toxicity made this trial significant. A phase III trial, RTOG 0522, evaluating cetuximab and radiotherapy against the standard of care platinum-based chemoradiotherapy is ongoing [58]. Clinical trials evaluating the fully humanized EGFR antibody, panitumimab, are also ongoing.

Another class of EGFR-targeted therapy includes the tyrosine kinase inhibitors (TKIs). Gefitinib and erlotonib are orally available TKIs that have shown clinical activity in a variety of SCCHN treatment settings [59,60]. Caponigro et al. summarizes recent clinical trials for this category of targeted therapies [61].

In general, EGFR-targeted therapies have low efficacy as monotherapies (cetuximab has about a $10 \%$ response rate when used alone), and thus are commonly administered 
concurrently with standard cytotoxic chemotherapy and/or radiotherapy. The limitations of delivering EGFR targeted therapy as a single agent may be due to persistent activation of signaling pathways downstream of EGFR. Co-targeting of critical signaling molecules may enhance therapeutic effects. For example, combined use of TKIs with monoclonal antibodies targeting EGFR is being investigated. Additionally, erlotonib and bevacizumab, a monoclonal antibody against vascular endothelial growth factor (VGEF) has shown promise.

3.2.5. COX-2-Keratinocyte inflammation has been shown to be crucial for chemical carcinogenesis especially in oral SCCHN. Various inflammatory mediators such as prostaglandins, interleukin-1, interleukin-6, and tumor necrosis factor $a$ are central to this process. Release of prostaglandins can induce vasodilation, changes in vascular permeability and inflammatory cell infiltration -all potential tumor promoting effects.

Cyclooxygenase-2 (COX-2) is an enzyme that catalyzes fatty acid oxygenation to produce eicosanoids. Specifically, it converts arachidonic acid to prostaglandin $\mathrm{H} 2$, which is further metabolized to thromboxanes and other prostaglandins (e.g., prostaglandin E2 or PGE2). COX-2 is overexpressed in many oral dysplasias and SCCHN tumors. PGE2 has been associated with malignant transformation in SCCHN [62]. COX-2 inhibitors have shown anti-proliferative effect and induce apoptosis in oral cancer cell lines. In vivo studies have shown reduction in PGE2 levels and dose-dependent reduction of tumor growth [63]. A recent phase II pilot trial failed to show chemopreventive efficacy of celecoxib in patients with premalignant oral lesions [64]. Clinical trials evaluating the efficacy of COX-2 inhibitors in the treatment of SCCHN are ongoing.

3.2.6. NF-kB-NF-kB (nuclear factor of kappa light polypeptide in B-cells), upon activation, translocates from the cytosol to the nucleus to induce transcription, leading to cell proliferation and reduced apoptosis. NF-kB is constitutively activated in many SCCHN tumors. NF-kB inhibitors include curcumin and proteasome inhibitors. A phase I trial is ongoing evaluating the proteasome inhibitor bortezomib in combination with cisplatin and radiotherapy for locoregionally advanced head and neck cancer for which no standard chemoradiation regimens exist (e.g., reirradiation) [65].

3.2.7. VEGF-The contribution of angiogenesis in the progression of solid tumors such as SCCHN is likely important. Without an adequate corresponding blood supply for a continually growing cancer, newly divided tumor cells would not survive without the necessary nutrients and oxygen. Vascular endothelial growth factor (VEGF) is a proangiogenic mediator that binds to VEGF receptors on endothelial cells. This results in endothelial migration, proliferation, and increased vascular permeability. High VEGF and VEGF receptor expression in patients with SCCHN are associated with higher tumor proliferation rates and worse survival [66]. A monoclonal antibody to VEGF, bevacizumab, has shown clinical efficacy in colorectal and renal cancers. In SCCHN, angiogenesis represents a potential mechanism of resistance against anti-EGFR agents. Vokes et al. conducted a phase I-II trial of erlotinib with bevacizumab in patients with metastatic and/or recurrent incurable SCCHN $[67,68]$. Favorable results were seen with no dose-limiting toxicities. Approximately $10 \%$ of patients had a complete or partial response while median progression-free survival was noted at 127 days. Investigators identified a biomarker, $\mathrm{pKDR} / \mathrm{KDR}$ ratio, that appeared to predict response to the combination therapy. Furthermore, the addition of bevacizumab to erlotinib resulted in increased inhibition of endothelial survival factors. Mature data are pending.

3.2.8. TGF- $\beta /$ Ras - Transforming growth factor- $\beta$ (TGF- $\beta$ ) plays a crucial role in cell differentiation, tissue regeneration, and regulation of the immune system. It was originally 
discovered to induce oncogenic transformation in rat kidney fibroblasts. TGF- $\beta$ receptors are single pass serine/threonine kinase receptors. The Ras genes were first associated with the transforming activities of the Harvey (H-ras) and Kirsten (K-ras) sarcoma viruses. Ras signal transduction pathways regulate cytoskeletal integrity, proliferation, differentiation, cell adhesion, apoptosis, and cell migration. Inappropriate Ras activation has been associated with malignant transformation. Ras mutations are rare in SCCHN in the western world ( $<5 \%$ ), while Ras overexpression is much more common [69]. Lu et al. studied the loss of transforming growth factor-b type II receptor (TGF $\beta$ RII) and its potential role in SCCHN tumorigenesis and progression. By combining this receptor loss with Ras overexpression (K-ras or H-ras) in a murine model, the investigators caused human SCCHN formation [70]. Neither alteration alone resulted in a malignant phenotype in previous studies. Ras overexpression is associated with hyperproliferation of malignant cells, but not malignant transformation itself in most studies. Evidence suggests that Ras may play some role in tumor initiation. TGF $\beta 1$, while having tumor-suppressive effects early, shows tumor progression effects in later stages.

\section{Molecular alterations in metastases of squamous cell carcinoma}

The presence or absence of cervical lymph node metastasis is the single most significant predictor of outcome in SCCHN. Involvement of the neck with malignancy reduces survival by $50 \%$ on average. Immunohistochemical profiling of primary and metastatic tumors has yielded reports on various genes implicated in metastasis. The fact that similar gene expression profiles are found between primary tumors and their metastases suggests that molecular alterations occur early in metastasis. Ecadherin is a cell adhesion molecule that regulates adhesion between adjacent epithelial cells. Downregulation of E-cadherin (and catenins) have been found in patients with metastatic SCCHN tumors [71]. Evidence suggests dissociation of tumor cells as a probable mechanism. Integrins are transmembrane proteins that participate in cell adhesion in addition to signal transduction. Increased cell motility and growth have been reported in head and neck cancer via an upregulation of integrin $a v \beta 6$ [72]. Overexpression of matrix metalloproteinase-2 (MMP2) and MMP9 have been associated with invasion, metastasis and poor prognosis [73]. Likewise, upregulation of EGFR, proangiogenic genes such as VEGF and IL-8, and chemokine receptor-7 (CCR7) have all been implicated in SCCHN metastasis [74]. Identification of tumors at high risk for regional and distant metastasis through molecular testing would have a profound impact on patient survival.

\section{Potential applications of molecular pathology in head and neck tumorigenesis}

\subsection{Early detection of HNSCC}

Diagnosing SCCHN tumors at the earliest possible stage is a critical component to improving outcomes. A recently developed oral rinse utilizes ELISA to detect soluble CD44, an overexpressed protein marker in SCCHN [75]. The assay detected elevated CD44 levels in $62 \%$ of the 102 SCCHN patients in the study group. In the control group of 69 patients with benign diseases of the upper aerodigestive tract, the test detected two patients with cancer before it was clinically evident. However, it also picked up a few false-positives. Sensitivity ranged from $62 \%$ to $70 \%$ while specificity ranged from $75 \%$ to $88 \%$. The authors combined the test for soluble CD44 with a test for CD44 promoter hypermethylation to increase sensitivity. 


\subsection{Biochemoprevention}

A large phase III trial evaluating 1190 early-stage SCCHN patients who were randomized to low-dose isotretinoin or placebo failed to show a reduction in the rate of second primary tumors in the treatment group [76]. Retinoids are still being studied in combination with other agents. An adjuvant biochemoprevention study included combination therapy with 13cis-retinoic acid (13-cRA), alpha-interferon, and alpha-tocopherol. This phase II trial included patients with advanced (stage III/IV) SCCHN and projected their 5-year diseasefree survival at $80 \%$. This approach unfortunately was abandoned after difficulty with patient accrual [77].

\subsection{Biomarkers in risk assessment and molecular staging}

5.3.1. Surgical margin test for risk of local recurrence-The adequacy of surgical resection/excision of SCCHN is routinely evaluated by frozen sections of margins. However, local recurrence is seen in up to $50 \%$ of patients with histologically benign margins [78]. Identification of pre-neoplastic molecular alterations in histologically tumorfree tissue will further improve margin evaluation. Gene promoter hypermethylation (e.g., p16, MGMT) is one of the more common mechanisms for loss of tumor suppressor gene function. Rosas et al. showed that at least $56 \%$ of SCCHN are characterized by abnormal methylation of p16, MGMT, or death-associated protein kinase [79]. The feasibility of intraoperative rapid DNA tissue extraction, rapid bisulfite treatment, and quantitative methylation-specific PCR (QMSP) for $p 16$ and $M G M T$ was studied by Goldenberg et al. [80]. While conventional QMSP requires about 24 hours, the rapid QMSP assay lasts 5 hours and requires simultaneous work of two individuals. This approach is potentially useful for cases requiring extensive resection and reconstruction.

5.3.2. Molecular predictors of survival-cDNA microarray technology has been utilized to predict outcomes in SCCHN patients. Belbin analyzed gene expression from 17 patients using gene chips containing 9,216 clones [81]. 375 differentially expressed genes were identified through this method enabling the investigators to categorize patients into two distinct groups. The favorable prognosis group had a 2-year disease-specific survival of $100 \%$, while the unfavorable group had a 56\% survival. This molecular assay was found to be superior in predicting outcome to standard clinicopathologic criteria. Genes identified in the unfavorable group included catalase, xanthine oxidase, cytokine family members, and phosphodiesterase genes.

5.3.3. MicroRNA-MicroRNA are endogenous $21-22$ nucleotide long non-protein-coding RNA sequences regulating target mRNA expression by complementary interaction with the $3^{\prime}$ untranslated region of mRNA. The extent of complementarity between microRNA and its target determines the mechanism of translational inhibition: partial complementarity will induce mRNA repression, while perfect complementarity will cause translational inhibition. MicroRNA biogenesis and its role in carcinogenesis are reviewed in by Gomes et al. [82].

A comparative study of 148 microRNAs in 18 cell lines of oral SCC and immortalized oral keratinocytes showed down-regulation of 54 microRNAs and up-regulation of 11 microRNA [83]. Although the mechanism of microRNA dysregulation in SCCHN remains largely unknown, down-regulation of 5 microRNAs in oral SCC is caused by $\mathrm{CpG}$ island hypermethylation [83]. Another survey of microRNA expression in nine SCCHN cell lines identified 54 commonly expressed microRNA genes and showed that the microRNA profile appears to be similar in all HNSCC tumors independent of anatomical head and neck subsites [84]. Two microRNA targets with known roles in HNSCC are identified - high mobility group A2 (HMGA2) controlled by microRNA-98 and pyruvate kinase type M2 
(PKM2) inhibited by microRNA-133 [85,86]. HMGA2 expression in oral SCC is an independent negative prognostic factor [86].

MicroRNA highly expressed by the cancer cells and undetectable in blood of healthy individuals may serve as novel tumor biomarkers. For instance, microRNA-184 was detected in $80 \%$ of cancer patients and in $13 \%$ of control individuals [85].

\section{Histopathology}

\subsection{Premalignant lesions of the head and neck}

A premalignant or precancerous epithelial lesion exhibits histological changes and has the increased potential of progressing to an invasive squamous cell carcinoma. These changes can include hyperplasia, and more ominously, mild to severe dysplasia. Clinical signs of premalignancy include leukoplakia and erythroplakia, which should not be used interchangeably with the histologic diagnoses.

6.1.1. Leukoplakia-Leukoplakia is a clinical term that describes a white patch or plaque that cannot be rubbed off and cannot be given a more specific clinical diagnosis. The prevalence of oral leukoplakia in the US is about $2.9 \%$, often affecting patients in their fifth to seventh decades of life [87]. Seventy to ninety percent of cases use tobacco, the only consistent etiologic factor [88]. Most clinical white patches are caused by chronic irritation (i.e., from tobacco or dental trauma) and, histologically, represent an irregularly thickened keratin layer varying from simple hyperparakeratosis to an early invasive SCCHN. As such, leukoplakia does not correlate well with the histologic diagnosis of dysplasia.

The potential for malignant transformation in oral leukoplakia is well documented. Two studies performed in the US observed an average transformation rate of $15.6 \%$ (range 13.6$17.5 \%$ ) with varying periods of observation $[89,90]$. Although malignancy is considered an irreversible state once it is achieved, studies have shown premalignancy to be reversible after cessation of the offending stimuli. In a prospective cohort of 12,212 Indian smokers, subjects who quit showed a significant decrease in the incidence of oral leukoplakia during follow-up (incidence ratio of 0.3). There was no decrease noted for patients with oral lichen planus [91]. Bouquot et al. found that nearly $80 \%$ of leukoplakic lesions regressed or disappeared within 12 months after smoking cessation [88].

DNA content within lesions containing leukoplakia has been found to be significant predictor of local recurrence following surgery. Patients with lesions harboring aneuploid or tetraploid DNA faired poorly compared to their normal diploid counterparts [92].

6.1.2. Erythroplakia-Erythroplakia is the "red" analogue of leukoplakia and is characterized by a velvety appearance with distinct borders. The incidence of erythroplakia is much lower than that of leukoplakia. However, it is associated more frequently with malignancy and is the most common clinical presentation of an early SCCHN. Roughly half of erythroplakic lesions may harbor invasive SCCHN with most, if not all, containing at least mild dysplasia. Although leukoplakia is associated with a significant spontaneous regression rate, erythroplakic lesions generally do not regress.

6.1.3. Squamous dysplasia-Squamous dysplasia implies that objective morphologic changes have occurred in epithelial cells. Dysplasia requires the association of architectural disorder with cytologic atypia. The architectural features of dysplasia include "drop-shaped" rete ridges, keratin pearls, irregular epithelial stratification, abnormally superficial mitoses, and loss of cell polarity [93]. The cytologic criteria used for the diagnosis and grading of dysplasia are hyperchromasia, variation in nuclear size, shape, increased nuclear- 
cytoplasmic ratio, atypical mitotic figures, location of mitotic figures, and dyskeratosis. The morphologic evaluation of dysplasia is similar throughout the upper aerodigestive tract. Biologically, squamous dysplasia represents a spectrum and no specific criteria exist to precisely divide this range of changes into mild, moderate, or severe categories. Most pathologists recognize the various degrees of dysplasia. However, numerous studies have reported a high degree of inter- and intra- observer variability in determining the presence and grading of squamous dysplasia [94-96]. Knowledge of the clinical scenario apparently does not improve agreement rates [97] and associated inflammation further decreases agreement rates [95]. Ulceration, irradiation, and some nutritional deficiencies (iron, folate, B12) can all simulate dysplastic changes.

A binary classification of squamous dysplasia has been considered; it is combining the "questionable or mild" dysplasia into a low risk group and "moderate to severe" dysplasia into a high risk group [98]. Decreasing the number of available choices from three to two improved agreement amongst pathologists. In mild and moderate dysplasia, the epithelial changes are limited to the lower third and middle third of the epithelium, respectively. In severe dysplasia and CIS, the changes approximate the full thickness. Severe dysplasia and CIS may be indistinguishable from each other and therefore should be treated in a similar fashion.

6.1.4. Lichen planus-The premalignant potential of oral lichen planus (LP) is controversial due to conflicting data in the literature, most of which is retrospective. One prospective study of 570 subjects with LP found malignant transformation in $1.2 \%$ with a mean follow-up of 5.6 years [99]. A more recent prospective study included patients both with LP (67 subjects) and oral lichenoid lesions (125 subjects). After a mean follow-up of 4.5 years, four patients with oral lichenoid lesions developed SCCHN while none with LP did. After comparing the expected against actual figures for number of subjects developing cancer, no increase in LP patients was found, while a 142-fold increase was identified in patients with oral lichenoid lesions $(p=0.04)$ [100].

6.1.5. Others-Submucous fibrosis is an idiopathic, precancerous condition that is rare in the US. The oral cavity and oropharynx are most commonly affected. Histologic features include epithelial atrophy, juxtaepithelial inflammation, and fibroelastic changes in the lamina propria. Rates of malignant transformation are reported between 8-33\%. Steroid and hyaluronidase injections, topical vitamin $\mathrm{A}$, and iron supplementation have not shown therapeutic benefit in this disease [101]. Sideropenic dysphagia occurs in patients with Plummer-Vinson syndrome (triad of postcricoid dysphagia, upper esophageal webs, and iron deficiency anemia) and is characterized by marked epithelial atrophy with increased turnover rates. The condition is strongly associated with esophageal cancer.

\subsection{Carcinoma in-situ (CIS) and early invasive squamous cell carcinoma}

The concept underlying carcinoma in-situ is that malignant transformation has already taken place, but invasion is absent. Technically, the lesion is no longer merely dysplastic at this point and is considered malignant. The histologic hallmark of malignancy is invasion. Invasion is easily recognized when cytologically abnormal squamous cells show perineural or vascular invasion and extend into subepithelial tissues. The evaluation for the earliest stages of invasion into the lamina propria is a common diagnostic problem. The loss of basement membrane material is not a reliable feature, as even an overtly invasive carcinoma can synthesize basement membrane-like material at its leading invasive edge. The histologic appearance of invasive SCCHN includes the following features: atypical squamous cells exhibiting nuclear atypia, increased nuclear-to-cytoplasmic ratio, varying degrees of keratinization (more with well-differentiated tumors, less with poorly-differentiated), 
intercellular bridges, increased and atypical mitotic figures; hyperchromasia, basement membrane invasion, and an associated inflammatory response. In the future, the molecular features described below will assist in routine evaluation of squamous dysplasia.

\section{Acknowledgments}

The authors of this work were supported by NIH grants T32 CA060397 and P50 CA097190 (to JRG) as well as an American Cancer Society Clinical Research Professorship (to JRG).

\section{References}

1. Jemal A, Siegel R, Ward R, et al. Cancer statistics. CA: A Cancer Journal for Clinicians. 2008; 58:71-96. [PubMed: 18287387]

2. Franceschi S, Bidoli E, Herrero R, Munoz N. Comparison of cancers of the oral cavity and pharynx worldwide: etiological clues. Oral Oncology. 2000; 36:106-115. [PubMed: 10889929]

3. FAO. Projections of tobacco production, consumption and trade to the year 2010; Rome, Italy. 2003. p. 11

4. Sturgis EM, Cinciripini PM. Trends in head and neck cancer incidence in relation to smoking prevalence: an emerging epidemic of human papillomavirus-associated cancers? Cancer. 2007; 110:1429-1435. [PubMed: 17724670]

5. Kinzler KW, Vogelstein B. Lessons from hereditary colorectal cancer. Cell. 1996; 87:159-170. [PubMed: 8861899]

6. Slaughter DP, Southwick DP, Smejkal W. Field cancerization in oral stratified squamous epithelium; clinical implications of multicentric origin. Cancer. 1953; 6:963-968. [PubMed: 13094644]

7. Califano J, van der Riet P, Westra W, et al. Genetic progression model for head and neck cancer: implications for field cancerization. Cancer Research. 1996; 56:2488-2492. [PubMed: 8653682]

8. Ha PK, Benoit PK, Yochem R, et al. A transcriptional progression model for head and neck cancer. Clin Cancer Res. 2003; 9:3058-3064. [PubMed: 12912957]

9. Weber RG, Scheer M, Born IA, et al. Recurrent chromosomal imbalances detected in biopsy material from oral premalignant and malignant lesions by combined tissue microdissection, universal DNA amplification, and comparative genomic hybridization. The American Journal of Pathology. 1998; 153:295-303. [PubMed: 9665491]

10. Brieger J, Jacob R, Riazimand HS, et al. Chromosomal aberrations in premalignant and malignant squamous epithelium. Cancer Genetics and Cytogenetics. 2003; 144:148-155. [PubMed: 12850378]

11. Kim J, Yook JI, Lee EH, et al. Evaluation of premalignant potential in oral lichen planus using interphase cytogenetics. Journal of Oral Pathology \& Medicine: Official Publication of the International Association of Oral Pathologists and the American Academy of Oral Pathology. 2001; 30:65-72.

12. Zuo C, Zhang H, Spencer HJ, et al. Increased microsatellite instability and epigenetic inactivation of the hMLH1 gene in head and neck squamous cell carcinoma. Otolaryngol Head Neck Surg. 2009; 141:484-490. [PubMed: 19786217]

13. Ha PK, Pilkington TA, Westra WH, Sciubba J, Sidransky D, Califano JA. Progression of microsatellite instability from premalignant lesions to tumors of the head and neck. International Journal of Cancer Journal International du Cancer. 2002; 102:615-617. [PubMed: 12448003]

14. El-Naggar AK, Hurr K, Huff V, Clayman GL, Luna MA, Batsakis JG. Microsatellite instability in preinvasive and invasive head and neck squamous carcinoma. The American Journal of Pathology. 1996; 148:2067-2072. [PubMed: 8669490]

15. Partridge M, Emilion G, Pateromichelakis S, A’Hern R, Phillips E, Langdon J. Allelic imbalance at chromosomal loci implicated in the pathogenesis of oral precancer, cumulative loss and its relationship with progression to cancer. Oral oncology. 1998; 34:77-83. [PubMed: 9682768] 
16. Mao L, Lee JS, Fan YH, et al. Frequent microsatellite alterations at chromosomes 9p21 and 3p14 in oral premalignant lesions and their value in cancer risk assessment. Nature medicine. 1996; 2:682-685.

17. Rosin MP, Cheng X, Poh C, et al. Use of allelic loss to predict malignant risk for low-grade oral epithelial dysplasia. Clinical cancer research: an official journal of the American Association for Cancer Research. 2000; 6:357-362. [PubMed: 10690511]

18. Zhang L, Rosin MP. Loss of heterozygosity: a potential tool in management of oral premalignant lesions? Journal of oral pathology \& medicine: official publication of the International Association of Oral Pathologists and the American Academy of Oral Pathology. 2001; 30:513-520.

19. Shin DM, Charuruks N, Lippman SM, et al. p53 protein accumulation and genomic instability in head and neck multistep tumorigenesis. Cancer epidemiology, biomarkers \& prevention: a publication of the American Association for Cancer Research, cosponsored by the American Society of Preventive Oncology. 2001; 10:603-609.

20. Cruz IB, Snijders PJ, Meijer CJ, et al. p53 expression above the basal cell layer in oral mucosa is an early event of malignant transformation and has predictive value for developing oral squamous cell carcinoma. The Journal of pathology. 1998; 184:360-368. [PubMed: 9664901]

21. Ogden GR, Chisholm DM, Morris AM, Stevenson JH. Overexpression of p53 in normal oral mucosa of oral cancer patients does not necessarily predict further malignant disease. The Journal of pathology. 1997; 182:180-184. [PubMed: 9274528]

22. Patel MM, Parekh LJ, Jha FP, et al. Clinical usefulness of telomerase activation and telomere length in head and neck cancer. Head \& neck. 2002; 24:1060-1067. [PubMed: 12454944]

23. Liao J, Mitsuyasu T, Yamane K, Ohishi M. Telomerase activity in oral and maxillofacial tumors. Oral oncology. 2000; 36:347-352. [PubMed: 10899673]

24. Zhang S, Dong S, Teng X, Chen T. Quantitative assay of telomerase activity in head and neck squamous cell carcinoma and other tissues. Archives of otolaryngology-head \& neck surgery. 2001; 127:581-585. [PubMed: 11346437]

25. Sumida T, Hamakawa H, Sogawa K, Sugita K, Tanioka K, Ueda K. Telomerase components as a diagnostic tool in human oral lesions. International journal of cancer Journal international du cancer. 1999; 80:1-4. [PubMed: 9935220]

26. Soni S, Kaur J, Kumar J, et al. Alterations of rb pathway components are frequent events in patients with oral epithelial dysplasia and predict clinical outcome in patients with squamous cell carcinoma. Oncology. 2005; 68:314-325. [PubMed: 16020958]

27. Yarbrough WG. The ARF-p16 gene locus in carcinogenesis and therapy of head and neck squamous cell carcinoma. The Laryngoscope. 2002; 112:2114-2128. [PubMed: 12461329]

28. Ha PK, Tong BC, Westra WH, et al. Mitochondrial C-tract alteration in premalignant lesions of the head and neck: a marker for progression and clonal proliferation. Clinical cancer research: an official journal of the American Association for Cancer Research. 2002; 8:2260-2265. [PubMed: 12114429]

29. Rubin Grandis J, Tweardy DJ, Melhem MF. Asynchronous modulation of transforming growth factor alpha and epidermal growth factor receptor protein expression in progression of premalignant lesions to head and neck squamous cell carcinoma. Clinical cancer research: an official journal of the American Association for Cancer Research. 1998; 4:13-20. [PubMed: 9516947]

30. Grandis JR, Drenning SD, Zeng Q, et al. Constitutive activation of Stat3 signaling abrogates apoptosis in squamous cell carcinogenesis in vivo. Proceedings of the National Academy of Sciences of the United States of America. 2000; 97:4227-4232. [PubMed: 10760290]

31. Srinivasan M, Jewell SD. Evaluation of TGF-alpha and EGFR expression in oral leukoplakia and oral submucous fibrosis by quantitative immunohistochemistry. Oncology. 2001; 61:284-292. [PubMed: 11721175]

32. Gollin SM. Chromosomal alterations in squamous cell carcinomas of the head and neck: window to the biology of disease. Head \& neck. 2001; 23:238-253. [PubMed: 11428456]

33. Patmore HS, Ashman JNE, Stafford ND, et al. Genetic analysis of head and neck squamous cell carcinoma using comparative genomic hybridisation identifies specific aberrations associated with laryngeal origin. Cancer letters. 2007; 258:55-62. [PubMed: 17920192] 
34. Bockmuhl U, Schluns K, Kuchler I, Petersen S, Petersen I. Genetic imbalances with impact on survival in head and neck cancer patients. The American journal of pathology. 2000; 157:369-375. [PubMed: 10934141]

35. Pearlstein RP, Benninger MS, Carey TE, et al. Loss of 18q predicts poor survival of patients with squamous cell carcinoma of the head and neck. Genes Chromosomes Cancer. 1998; 21:333-339. [PubMed: 9559345]

36. Juhasz A, Balazs M, Sziklay I, et al. Chromosomal imbalances in laryngeal and hypopharyngeal cancers detected by comparative genomic hybridization. Cytometry Part A: the journal of the International Society for Analytical Cytology. 2005; 67:151-160. [PubMed: 16163698]

37. Nordkvist A, Gustafsson H, Juberg-Ode M, Stenman G. Recurrent rearrangements of 11q14-22 in mucoepidermoid carcinoma. Cancer genetics and cytogenetics. 1994; 74:77-83. [PubMed: 8019965]

38. Tonon G, Modi S, Wu L, et al. t(11;19)(q21;p13) translocation in mucoepidermoid carcinoma creates a novel fusion product that disrupts a Notch signaling pathway. Nat Genet. 2003; 33:208213. [PubMed: 12539049]

39. Tirado Y, Williams MD, Hanna EY, Kaye FJ, Batsakis JG, El-Naggar AK. CRTC1/MAML2 fusion transcript in high grade mucoepidermoid carcinomas of salivary and thyroid glands and Warthin's tumors: implications for histogenesis and biologic behavior. Genes Chromosomes Cancer. 2007; 46:708-715. [PubMed: 17437281]

40. Jin YT, Kayser S, Kemp BL, et al. The prognostic significance of the biomarkers p21WAF1/CIP1, p53, and bcl-2 in laryngeal squamous cell carcinoma. Cancer. 1998; 82:2159-2165. [PubMed: 9610695]

41. Narayana A, Vaughan AT, Gunaratne S, Kathuria S, Walter SA, Reddy SP. Is p53 an independent prognostic factor in patients with laryngeal carcinoma? Cancer. 1998; 82:286-291. [PubMed: 9445184]

42. Erber R, Conradt C, Homann N, et al. TP53 DNA contact mutations are selectively associated with allelic loss and have a strong clinical impact in head and neck cancer. Oncogene. 1998; 16:16711679. [PubMed: 9582015]

43. Mineta H, Borg A, Dictor M, Wahlberg P, Akervall J, Wennerberg J. p53 mutation, but not p53 overexpression, correlates with survival in head and neck squamous cell carcinoma. British Journal of Cancer. 1998; 78:1084-1090. [PubMed: 9792155]

44. Nagai MA, Miracca EC, Yamamoto L, et al. TP53 genetic alterations in head-and-neck carcinomas from Brazil. International Journal of Cancer Journal International du Cancer. 1998; 76:13-18. [PubMed: 9533755]

45. Hegde PU, Brenski AC, Caldarelli DD, et al. Tumor angiogenesis and p53 mutations: prognosis in head and neck cancer. Archives of Otolaryngology-Head \& Neck Surgery. 1998; 124:80-85. [PubMed: 9440785]

46. Poeta ML, Manola J, Goldwasser MA, et al. TP53 mutations and survival in squamous-cell carcinoma of the head and neck. The New England Journal of Medicine. 2007; 357:2552-2561. [PubMed: 18094376]

47. Shin DM, Mao L, Papadimitrakopoulou VM, et al. Biochemopreventive therapy for patients with premalignant lesions of the head and neck and p53 gene expression. Journal of the National Cancer Institute. 2000; 92:69-73. [PubMed: 10620636]

48. Clayman GL, el-Naggar AK, Lippman SM, et al. Adenovirus-mediated p53 gene transfer in patients with advanced recurrent head and neck squamous cell carcinoma. J Clin Oncol. 1998; 16:2221-2232. [PubMed: 9626224]

49. Lee B-J, Wang S-G, Choi J-S, Lee J-C, Goh E-K, Kim M-G. The prognostic value of telomerase expression in peripheral blood mononuclear cells of head and neck cancer patients. American Journal of Clinical Oncology. 2006; 29:163-167. [PubMed: 16601436]

50. Thurnher D, Knerer B, Formanek M, Kornfehl J. Non-radioactive semiquantitative testing for the expression levels of telomerase activity in head and neck squamous cell carcinomas may be indicative for biological tumour behaviour. Acta Oto-Laryngologica. 1998; 118:423-427. [PubMed: 9655221] 
51. Ernster JA, Sciotto CG, O’Brien MM, Robinson LJ, Willson T. Prevalence of oncogenic human papillomavirus 16 and 18 in the palatine tonsils of the general adult population. Arch Otolaryngol Head Neck Surg. 2009; 135:554-557. [PubMed: 19528402]

52. Bouda M, Gorgoulis VG, Kastrinakis NG, et al. "High risk" HPV types are frequently detected in potentially malignant and malignant oral lesions, but not in normal oral mucosa. Mod Pathol. 2000; 13:644-653. [PubMed: 10874669]

53. Gillison ML, Shah KV. Human papillomavirus-associated head and neck squamous cell carcinoma: mounting evidence for an etiologic role for human papillomavirus in a subset of head and neck cancers. Current Opinion in Oncology. 2001; 13:183-188. [PubMed: 11307062]

54. Herbst RS, Arquette M, Shin DM, et al. Phase II multicenter study of the epidermal growth factor receptor antibody cetuximab and cisplatin for recurrent and refractory squamous cell carcinoma of the head and neck. J Clin Oncol. 2005; 23:5578-5587. [PubMed: 16009949]

55. Baselga J, Trigo JM, Bourhis J, et al. Phase II multicenter study of the antiepidermal growth factor receptor monoclonal antibody cetuximab in combination with platinum-based chemotherapy in patients with platinum-refractory metastatic and/or recurrent squamous cell carcinoma of the head and neck. J Clin Oncol. 2005; 23:5568-5577. [PubMed: 16009950]

56. Burtness B, Goldwasser MA, Flood W, Mattar B, Forastiere AA. Phase III randomized trial of cisplatin plus placebo compared with cisplatin plus cetuximab in metastatic/recurrent head and neck cancer: an Eastern Cooperative Oncology Group study. J Clin Oncol. 2005; 23:8646-8654. [PubMed: 16314626]

57. Bonner JA, Harari PM, Giralt J, et al. Radiotherapy plus cetuximab for squamous-cell carcinoma of the head and neck. N Engl J Med. 2006; 354:567-578. [PubMed: 16467544]

58. Haddad RI, Shin DM. Recent advances in head and neck cancer. The New England Journal of Medicine. 2008; 359:1143-1154. [PubMed: 18784104]

59. Cohen EE, Kane MA, List MA, et al. Phase II trial of gefitinib $250 \mathrm{mg}$ daily in patients with recurrent and/or metastatic squamous cell carcinoma of the head and neck. Clinical Cancer Research: An Official Journal of the American Association for Cancer Research. 2005; 11:84188424. [PubMed: 16322304]

60. Soulieres D, Senzer NN, Vokes EE, Hidalgo M, Agarwala SS, Siu LL. Multicenter phase II study of erlotinib, an oral epidermal growth factor receptor tyrosine kinase inhibitor, in patients with recurrent or metastatic squamous cell cancer of the head and neck. J Clin Oncol. 2004; 22:77-85. [PubMed: 14701768]

61. Caponigro F, Milano A, Basile M, Ionna F, Iaffaioli RV. Recent advances in head and neck cancer therapy: the role of new cytotoxic and molecular-targeted agents. Current Opinion in Oncology. 2006; 18:247-252. [PubMed: 16552236]

62. Sumitani K, Kamijo R, Toyoshima et al T. Specific inhibition of cyclooxygenase-2 results in inhibition of proliferation of oral cancer cell lines via suppression of prostaglandin E2 production. Journal of Oral Pathology \& Medicine: Official Publication of the International Association of Oral Pathologists and the American Academy of Oral Pathology. 2001; 30:41-47.

63. Zweifel BS, Davis BS, Ornberg RL, Masferrer JL. Direct evidence for a role of cyclooxygenase 2derived prostaglandin E2 in human head and neck xenograft tumors. Cancer Res. 2002; 62:67066711. [PubMed: 12438270]

64. Papadimitrakopoulou JL, William WN Jr, Dannenberg AJ, et al. Pilot randomized phase II study of celecoxib in oral premalignant lesions. Clinical Cancer Research: An Official Journal of the American Association for Cancer Research. 2008; 14:2095-2101. [PubMed: 18381950]

65. Kubicek GJ, Machtay M, Axelrod M, et al. Phase I trial of bortezomib (VELCADE), cisplatin and radiotherapy for advanced head and neck cancer. J Clin Oncol. 2008; 26:abstract 6028.

66. Caponigro F, Formato F, Caraglia F, Normanno F, Iaffaioli RV. Monoclonal antibodies targeting epidermal growth factor receptor and vascular endothelial growth factor with a focus on head and neck tumors. Current Opinion in Oncology. 2005; 17:212-217. [PubMed: 15818163]

67. Vokes RV, Cohen EEW, Mauer AM, et al. A phase I study of erlotinib and bevacizumab for recurrent or metastatic squamous cell carcinoma of the head and neck (HNC). J Clin Oncol. 2005; 23:Abstract 5504. 
68. Seiwert TY, Davis DW, Yan D, et al. pKDR/KDR ratio predicts response in a phase I/II pharmacodynamic study of erlotinib and bevacizumab for recurrent or metastatic head and neck cancer (HNC). J Clin Oncol. 2007; 25:Abstract 6021.

69. Hoa M, Davis SL, Ames SJ, Spanjaard RA. Amplification of wild-type K-ras promotes growth of head and neck squamous cell carcinoma. Cancer Research. 2002; 62:7154-7156. [PubMed: 12499248]

70. Lu SL, Herrington H, Reh D, et al. Loss of transforming growth factor-beta type II receptor promotes metastatic head-and-neck squamous cell carcinoma. Genes Dev. 2006; 20:1331-1342. [PubMed: 16702406]

71. Tanaka N, Odajima T, Ogi K, Ikeda K, Satoh M. Expression of E-cadherin, alpha-catenin, and beta-catenin in the process of lymph node metastasis in oral squamous cell carcinoma. British Journal of Cancer. 2003; 89:557-563. [PubMed: 12888830]

72. Shintani S, Li C, Mihara M, Nakashiro K, Hamakawa H. Gefitinib ('Iressa'), an epidermal growth factor receptor tyrosine kinase inhibitor, mediates the inhibition of lymph node metastasis in oral cancer cells. Cancer Letters. 2003; 201:149-155. [PubMed: 14607328]

73. Kawata R, Shimada T, Maruyama S, Hisa S, Takenaka S, Murakami Y. Enhanced production of matrix metalloproteinase-2 in human head and neck carcinomas is correlated with lymph node metastasis. Acta Oto-Laryngologica. 2002; 122:101-106. [PubMed: 11876588]

74. Howell GM, Grandis JR. Molecular mediators of metastasis in head and neck squamous cell carcinoma. Head \& Neck. 2005; 27:710-717. [PubMed: 15952195]

75. Franzmann EJ, Reategui EP, Pedroso F, et al. Soluble CD44 is a potential marker for the early detection of head and neck cancer. Cancer Epidemiology, Biomarkers \& Prevention: A Publication of the American Association for Cancer Research, Cosponsored by the American Society of Preventive Oncology. 2007; 16:1348-1355.

76. Khuri FR, Lee FR, Lippman SM, et al. Randomized phase III trial of low-dose isotretinoin for prevention of second primary tumors in stage I and II head and neck cancer patients. Journal of the National Cancer Institute. 2006; 98:441-450. [PubMed: 16595780]

77. Shin DM, Khuri FR, Murphy B, et al. Combined interferon-alfa, 13-cis-retinoic acid, and alphatocopherol in locally advanced head and neck squamous cell carcinoma: novel bioadjuvant phase II trial. J Clin Oncol. 2001; 19:3010-3017. [PubMed: 11408495]

78. Batsakis JG. Surgical excision margins: a pathologist's perspective. Adv Anat Pathol. 1999; 6:140-148. [PubMed: 10342011]

79. Rosas SL, Koch W, da Costa Carvalho MG, et al. Promoter hypermethylation patterns of p16, O6methylguanine-DNA-methyltransferase, and death-associated protein kinase in tumors and saliva of head and neck cancer patients. Cancer Research. 2001; 61:939-942. [PubMed: 11221887]

80. Goldenberg D, Harden S, Masayesva BG, et al. Intra-operative molecular margin analysis in head and neck cancer. Archives of Otolaryngology-Head \& Neck Surgery. 2004; 130:39-44. [PubMed: 14732766]

81. Belbin TJ, Singh B, Barber B, et al. Molecular classification of head and neck squamous cell carcinoma using cDNA microarrays. Cancer Research. 2002; 62:1184-1190. [PubMed: 11861402]

82. Gomes CC, Gomez CC. MicroRNA and oral cancer: future perspectives. Oral Oncol. 2008; 44:910-914. [PubMed: 18620891]

83. Kozaki K, Imoto I, Mogi S, Omura K, Inazawa K. Exploration of tumor-suppressive microRNAs silenced by DNA hypermethylation in oral cancer. Cancer Res. 2008; 68:2094-2105. [PubMed: 18381414]

84. Tran N, McLean T, Zhang X, et al. MicroRNA expression profiles in head and neck cancer cell lines. Biochem Biophys Res Commun. 2007; 358:12-17. [PubMed: 17475218]

85. Wong TS, Liu XB, Wong BY, Ng RW, Yuen AP, Wei WI. Mature miR-184 as Potential Oncogenic microR-NA of Squamous Cell Carcinoma of Tongue. Clin Cancer Res. 2008; 14:25882592. [PubMed: 18451220]

86. Miyazawa J, Mitoro A, Kawashiri A, Chada KK, Imai KK. Expression of mesenchyme-specific gene HMGA2 in squamous cell carcinomas of the oral cavity. Cancer Res. 2004; 64:2024-2029. [PubMed: 15026339] 
87. Bouquot JE, Gorlin RJ. Leukoplakia, lichen planus, and other oral keratoses in 23,616 white Americans over the age of 35 years. Oral Surgery, Oral Medicine, and Oral Pathology. 1986; 61:373-381.

88. Bouquot JE, Whitaker SB. Oral leukoplakia-rationale for diagnosis and prognosis of its clinical subtypes or "phases". Quintessence International (Berlin, Germany: 1985). 1994; 25:133-140.

89. Silverman S, Gorsky S, Lozada F. Oral leukoplakia and malignant transformation. A follow-up study of 257 patients. Cancer. 1984; 53:563-568. [PubMed: 6537892]

90. Lumerman H, Freedman H, Kerpel S. Oral epithelial dysplasia and the development of invasive squamous cell carcinoma. Oral Surgery Oral Medicine Oral Pathology Oral Radiology and Endodontics. 1995; 79:321-329.

91. Gupta PC, Murti PR, Bhonsle RB, Mehta FS, Pindborg JJ. Effect of cessation of tobacco use on the incidence of oral mucosal lesions in a 10-yr follow-up study of 12,212 users. Oral Diseases. 1995; 1:54-58. [PubMed: 7553382]

92. Sudbo J, Kildal W, Risberg B, Koppang HS, Danielsen HE, Reith A. DNA content as a prognostic marker in patients with oral leukoplakia. The New England Journal of Medicine. 2001; 344:1270 1278. [PubMed: 11320386]

93. Pindborg, JJ.; Wahi, PN. Histological Typing of Cancer and Precancer of the Oral Mucosa. 2. Berlin; New York: Springer; 1997.

94. Tabor MP, Braakhuis BJM, van der Wal JE, et al. Comparative molecular and histological grading of epithelial dysplasia of the oral cavity and the oropharynx. The Journal of Pathology. 2003; 199:354-360. [PubMed: 12579537]

95. Fischer DJ, Epstein JB, Morton TH, Schwartz SM. Interobserver reliability in the histopathologic diagnosis of oral pre-malignant and malignant lesions. Journal of Oral Pathology \& Medicine: Official Publication of the International Association of Oral Pathologists and the American Academy of Oral Pathology. 2004; 33:65-70.

96. Kujan O, Khattab A, Oliver RJ, Roberts SA, Thakker N, Sloan N. Why oral histopathology suffers inter-observer variability on grading oral epithelial dysplasia: an attempt to understand the sources of variation. Oral Oncology. 2007; 43:224-231. [PubMed: 16931119]

97. Abbey LM, Kaugars GE, Gunsolley GE, et al. The effect of clinical information on the histopathologic diagnosis of oral epithelial dysplasia. Oral Surgery, Oral Medicine, Oral Pathology, Oral Radiology, and Endodontics. 1998; 85:74-77.

98. Kujan O, Oliver RJ, Khattab A, Roberts SA, Thakker N, Sloan P. Evaluation of a new binary system of grading oral epithelial dysplasia for prediction of malignant transformation. Oral Oncology. 2006; 42:987-993. [PubMed: 16731030]

99. Silverman S, Gorsky M, Lozada-Nur F. A prospective follow-up study of 570 patients with oral lichen planus: persistence, remission, and malignant association. Oral Surgery Oral Medicine and Oral Pathology. 1985; 60:30-34.

100. van der Meij EH, Mast H, van der Waal I. The possible premalignant character of oral lichen planus and oral lichenoid lesions: a prospective five-year follow-up study of 192 patients. Oral Oncology. 2007; 43:742-748. [PubMed: 17112770]

101. Barnes, L. Surgical Pathology of the Head and Neck. 3. New York: Informa Healthcare; 2008. 
EGFR, STAT3

Normal mucosa

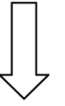

Hyperplasia

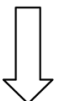

Mild dysplasia

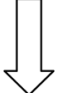

Moderate dysplasia

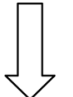

Severe dysplasia

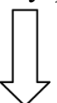

CIS

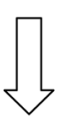

Invasive SCC

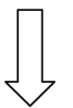

Metastasis

p16/9p21

$\mathrm{Rb}$

telomerase

p53

HPV

LOH 9p21/p16

LOH 3p14/FHIT

3q, 8q gains/MYC

cyclin D1

$\mathrm{NF}-\mathrm{kB}$

TGF- $\beta$

VEGF

MMP

Fig. 1.

Molecular alterations associated with various stages of head and neck carcinogenesis. Genes listed are not necessarily in order of pathogenesis. 